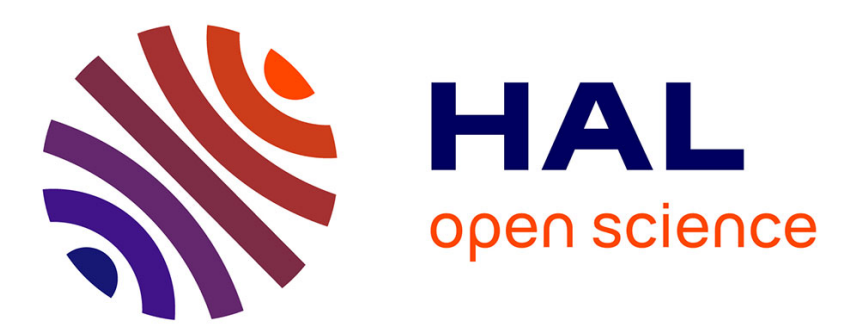

\title{
Three-Wave Trapponic Solitons for Tunable High-Repetition Rate Pulse Train Generation
}

Fabio Baronio, Matteo Conforti, Antonio Degasperis, Stefan Wabnitz

\section{To cite this version:}

Fabio Baronio, Matteo Conforti, Antonio Degasperis, Stefan Wabnitz. Three-Wave Trapponic Solitons for Tunable High-Repetition Rate Pulse Train Generation. IEEE Journal of Quantum Electronics, 2008, 44 (6), pp.542-546. 10.1109/JQE.2008.917970 . hal-02397772

\section{HAL Id: hal-02397772 \\ https://hal.science/hal-02397772}

Submitted on 6 Dec 2019

HAL is a multi-disciplinary open access archive for the deposit and dissemination of scientific research documents, whether they are published or not. The documents may come from teaching and research institutions in France or abroad, or from public or private research centers.
L'archive ouverte pluridisciplinaire HAL, est destinée au dépôt et à la diffusion de documents scientifiques de niveau recherche, publiés ou non, émanant des établissements d'enseignement et de recherche français ou étrangers, des laboratoires publics ou privés. 


\title{
Three-wave trapponic solitons for tunable high-repetition rate pulse train generation
}

\author{
Fabio Baronio, Member, IEEE, Matteo Conforti, Antonio Degasperis, and Stefan Wabnitz, Member, IEEE
}

\begin{abstract}
We demonstrate that the parametric mixing of an ultra-short Zakharov-Manakov (ZM) soliton pulse with a quasicontinuous wave $(\mathrm{CW})$ signal in a weakly quadratic nonlinear and dispersive medium may lead to the generation of a three-wave soliton with variable speed, or trappon. We show that trappons may provide a novel mechanism of cavity-less ultra-short pulse train generation. The time width, the amplitude and the repetition rate of the generated pulse trains may be controlled in a stable manner by adjusting the intensity level of the CW signal and/or the ZM short soliton pulse.
\end{abstract}

Index Terms-Three wave interaction, Solitons, Pulse train generation.

\section{INTRODUCTION}

$\mathbf{E}$ FFICIENT mechanisms for the generation of highcontrast, ultra-short pulse trains are of interest in a broad range of domains, such as time-resolved spectroscopy and microscopy, selective femtosecond chemistry, quantum coherent control in high-field physics, and optical communications. Relevant application examples include quantumpath control of high-harmonic generation [1]; multi-pulse excitation of atoms, molecules, and solids [2]; multiple-laserpulse excitation of high-gradient plasma accelerators [3]; high-fluence $\mathrm{THz}$ wave-train generation for radar, microwave [4] and optical communication systems. Several techniques for generating trains of ultra-short pulses at repetition rates beyond those achievable by active laser mode locking or by means of electrically-controlled modulation have been explored in recent years. For example, linear pulse train generation techniques include the repetition-rate multiplication of a lower rate source by applying amplitude [5], [6] or phasespectral filtering [7], [8], [9]. Moreover, different all-optical techniques that may result in up to $\mathrm{THz}$ pulse train rates have also been proposed by using both quadratic and cubic nonlinear media. Consider for example induced modulation instability [10], [11], multiple four-wave-mixing [12], [13], and backward quasi-phase-matched second harmonic generation [14], [15].

In this Paper, we present a novel nonlinear technique for the flexible generation of a train of ultra-short optical pulses

F. Baronio and M. Conforti are with the Dipartimento di Elettronica per l'Automazione, Università di Brescia, via Branze 38, Brescia 25123, Italy.

A. Degasperis is with the Dipartimento di Fisica, Università di Roma "La Sapienza", and Istituto Nazionale di Fisica Nucleare, P.le A. Moro 2, 00185 Roma, Italy.

S. Wabnitz was with the Institut Carnot de Bourgogne, UMR 5209 CNRS, Université de Bourgogne, 9 Av. A. Savary BP 46870, 21078 Dijon, France. His present addres is Dipartimento di Elettronica per l'Automazione, Università di Brescia, via Branze 38, Brescia 25123, Italy. with light-controlled time width, amplitude and repetition rate. This technique originates from the interaction of three waves at resonance. Though this process is of interest in various physical contexts, our treatment here is phrased in the language of nonlinear optics where its potential applicability appears to be higher. The physical model describing threewave interactions (TWI) is completely integrable by spectral methods [16], and it exhibits soliton solutions, i.e. particular waveforms that preserve their shapes upon collisions. The simplest of such soliton solutions of the TWI equations was discovered by Zakharov and Manakov (ZM), and it consists of hyperbolic secant pulses. We propose to exploit the parametric interaction of an ultra-short ZM soliton pulse [16] with a quasi-continuous wave signal, which collide in a weakly dispersive and nonlinear quadratic optical crystal. As we shall see, the phase-matched three-wave interaction of the ultrashort pulse with the $\mathrm{CW}$ signal may lead to the high-contrast modulation of the $\mathrm{CW}$ signal into a sequence of ultra-short pulses. Quite remarkably, the above pulse train generation process is analytically described in terms of the adiabatic reshaping of the initial single-wave ZM soliton into a threewave locked soliton with variable speed, which is also known as the trappon [17]. By exploiting the degrees of freedom of such trapponic soliton solutions, we shall demonstrate here that the time width, amplitude and repetition rate of the generated pulse train may be controlled in a stable manner by simply adjusting the parameters of the two input pulses, namely the intensity level of the CW signal, and/or the intensity level and time width of the short ZM soliton pulse.

\section{THREE-WAVE INTERACTION EQUATIONS}

The dimensionless equations that describe the quadratic resonant interaction of three waves in a nonlinear optical medium read as

$$
\begin{aligned}
& \frac{\partial A_{1}}{\partial \xi}+\delta_{1} \frac{\partial A_{1}}{\partial \tau}=i A_{2}^{*} A_{3}, \\
& \frac{\partial A_{2}}{\partial \xi}+\delta_{2} \frac{\partial A_{2}}{\partial \tau}=i A_{1}^{*} A_{3}, \\
& \frac{\partial A_{3}}{\partial \xi}+\delta_{3} \frac{\partial A_{3}}{\partial \tau}=i A_{1} A_{2},
\end{aligned}
$$

with

$$
A_{j}=\pi \chi^{(2)} \sqrt{\frac{n_{j} \omega_{1} \omega_{2} \omega_{3}}{n_{1} n_{2} n_{3} \omega_{j}}} E_{j} .
$$

Here $\tau=t / t_{0}, t_{0}$ is an arbitrary time scale; $\xi=z / z_{0}, z_{0}$ is an unit space-propagation parameter. $E_{j}$ is the slowly varying 
electric field envelope of the wave at frequency $\omega_{j}, n_{j}$ is its refractive index, $\chi^{(2)}$ is the quadratic nonlinear susceptibility, $\delta_{j}=z_{0} /\left(v_{j} t_{0}\right)\left(v_{j}\right.$ being the linear group velocity), and $j=$ $1,2,3$. We assume that the group velocity $v_{3}$ of the wave with the highest frequency $\left(\omega_{3}=\omega_{1}+\omega_{2}\right)$ lies between the group velocities of the other waves, i.e. $v_{1}>v_{3}>v_{2}$. With no loss of generality, we shall write Eqs. (1) in a coordinate system such that $\delta_{1}=0$, which implies $0<\delta_{3}<\delta_{2}$. Eqs. (1) exhibit, among others, the conserved quantities

$$
\begin{aligned}
& U_{13}=U_{1}+U_{3}=\frac{1}{2} \int_{-\infty}^{+\infty}\left(\left|A_{1}\right|^{2}+\left|A_{3}\right|^{2}\right) d \tau, \\
& U_{23}=U_{2}+U_{3}=\frac{1}{2} \int_{-\infty}^{+\infty}\left(\left|A_{2}\right|^{2}+\left|A_{3}\right|^{2}\right) d \tau,
\end{aligned}
$$

which is reported here for future reference, where $\mathbf{U}_{1}, U_{2}$ and $2 U_{3}$ represent the energies of waves at the frequencies $\omega_{1}, \omega_{2}$ and $\omega_{3}$, respectively.

\section{SOLITON-BASED ULTRA-SHORT PULSE TRAIN GENERATION}

Let us first present numerical evidence of the train generation process. Figure 1 illustrates the possibility of obtaining an efficient modulation of the input $\mathrm{CW}$ signal into a train of ultra-short pulses, as a result of its parametric mixing with an ultra-short ZM soliton pulse at a different carrier wavelength. In particular, in Fig. 1 we numerically solved Eqs. (1), where the initial excitation conditions at $\xi=0$ were provided by a quasi-CW signal at frequency $\omega_{1}$ (a standing signal in a coordinate system where $\delta_{1}=0$ ) and a short ZM soliton pulse at frequency $\omega_{2}$ (with delay parameter $\delta_{2}>0$ ). The two input pulses were pre-delayed, so that they could collide at some distance inside the crystal. Figure 1 shows that as soon as the faster ZM soliton pulse starts to overlap in time with the slower quasi-CW signal, their parametric mixing leads to the generation of an idler short pulse at frequency $\omega_{3}$. Additionally, Fig. 1 also shows that the TWI leads to a periodic oscillation with distance $\xi$ of the intensity of the two short pulses at carrier frequencies $\omega_{2}$ and $\omega_{3}$. As a result, it turns out [18] that the centre of mass of these two pulses periodically oscillates in time around an average value, which grows larger with distance according to a common average group velocity which is apparently (see Fig. 1) different from the group velocity of the incoming short pulse. As for the incoming CW signal, Fig. 1 also shows that a train of short pulses is carved on the $\mathrm{CW}$ background at frequency $\omega_{1}$. Finally, Fig. 1 also illustrates that at the end of the threewave interaction process the quasi-CW background at $\omega_{1}$ is modulated into a sequence of ultra-short pulses, the soliton pulse at $\omega_{2}$ returns back to its original shape, and the generated sum-frequency pulse at $\omega_{3}$ vanishes.

Quite remarkably, we shall demonstrate now that the entire three-wave interaction process which is displayed in Fig. 1 may be analytically represented in terms of particular analytical TWI soliton solutions. In the notation of Eqs. (1), the arbitrary input quasi $\mathrm{CW}$ signal at frequency $\omega_{1}$ may be described as
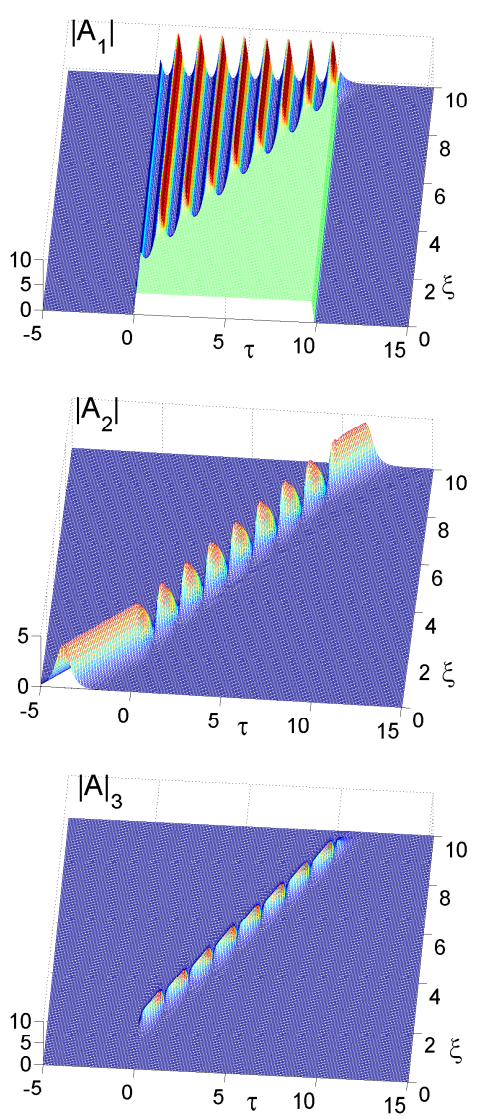

Fig. 1. Sum-frequency parametric interaction of a quasi-CW control at $\omega_{1}$ and a short pulse at $\omega_{2}$. The characteristic delays are $\delta_{1}=0, \delta_{2}=2, \delta_{3}=1$.

$$
\begin{aligned}
& A_{1}=\frac{C e^{-i \gamma \tau}}{2}\left[\tanh \left(\frac{\tau-\tau_{i}}{\tau_{r}}\right)-\tanh \left(\frac{\tau-\tau_{f}}{\tau_{r}}\right)\right], \\
& A_{2}=0 \\
& A_{3}=0
\end{aligned}
$$

where $C$ is the complex amplitude and $\gamma$ is the frequency shift with respect to $\omega_{1}$ of the quasi-CW signal; $\tau_{i}\left(\tau_{f}\right)$ and $\tau_{r}$ are the switch-on (switch-off) time and the rise/fall time of the quasi-CW signal, respectively.

On the other hand, the input ZM single-wave soliton pulse [16] at frequency $\omega_{2}$ reads as

$$
\begin{aligned}
& A_{1}=0, \\
& A_{2}=2 P \sqrt{\varrho \delta_{2} \delta_{3}} \frac{e^{i \varrho \phi \tau_{2}}}{\cosh \left(2 P \varrho \tau_{2}\right)}, \\
& A_{3}=0
\end{aligned}
$$

where

$$
\begin{aligned}
\varrho & =\delta_{3} /\left(\delta_{2}-\delta_{3}\right), \\
\tau_{2} & =-\tau+\delta_{2} \xi .
\end{aligned}
$$

For a given choice of the three linear group velocities, or characteristic delays $\delta_{j}$, the above ZM soliton is determined in terms of the two real parameters $P>0, \phi$. The parameter $P$ fixes both the soliton peak amplitude and its temporal width. 
Whereas the parameter $\phi$ corresponds to a phase shift which is linear in both $\tau$ and $\xi$.

After the collision of the $\mathrm{ZM}$ pulse with the $\mathrm{CW}$ background, we surmise that their parametric interaction generates a three-wave trapponic soliton, which consists of a groupvelocity locked bright-bright-dark triplet. Similar triplet solutions have been already considered in nonlinear optics in the form of simultons [19], [20] or boomerons [21]. Trappon soliton solutions of the TWI Eqs. (1) may be found as discussed in the recent analysis of Ref. [18], [19]. TWI trappons, whose potential impact on nonlinear optics is suggested here for the first time, are characterized by a periodic (or trapped) spatio-temporal evolution of the energy distribution of the three waves, as well as of their locked propagation speed. As a result, the center of mass of a trappon also periodically oscillates within a limited time interval. As far as the potential applications are concerned, the most significant property of the trappon dynamics consists in the carving of a train of short pulses into the CW-background at frequency $\omega_{1}$. Indeed, as shown in the next section, the entire three-wave interaction dynamics after the collision of the short pulse with the $\mathrm{CW}$ background as shown by Fig. 1 may be analytically described in terms of the trappon solution

$$
\begin{aligned}
A_{1}^{T} & =i a \sqrt{\delta_{2} \delta_{3}} e^{i q_{1} \tau_{1}}-i \frac{\Delta}{4 p}\left(\frac{\delta_{2}-\delta_{3}}{\delta_{2} \delta_{3}}\right) A_{2}^{*} A_{3}, \\
A_{2}^{T} & =-\frac{2 p \delta_{3}}{\Delta} \sqrt{\frac{2 \delta_{2}}{\delta_{2}-\delta_{3}}} e^{i q_{2} \tau_{2}}\left(H_{+}^{*}-H_{-}^{*}\right), \\
A_{3}^{T *} & =\frac{2 p \delta_{2}}{\Delta} \sqrt{\frac{2 \delta_{3}}{\delta_{2}-\delta_{3}}} e^{i q_{3} \tau_{3}}\left(H_{+} e^{i \beta}-H_{-} e^{-i \beta}\right),
\end{aligned}
$$

where

$$
\begin{aligned}
\Delta & =1+\left|H_{+}\right|^{2}+\left|H_{-}\right|^{2}-2 \cos (\beta) \mathcal{R} e\left(H_{+} H_{-}^{*} e^{i \beta}\right) \\
H_{ \pm} & =e^{-(B \pm i \chi) \tau} e^{\frac{2 \delta_{2} \delta_{3}}{\delta_{2}-\delta_{3}} p \xi} \\
\chi & =\sqrt{a^{2}-p^{2}} \\
B & =p\left(\frac{\delta_{2}+\delta_{3}}{\delta_{2}-\delta_{3}}\right), \tan (\beta)=\sqrt{a^{2}-p^{2}} / p \\
\delta & =\frac{2 \delta_{2} \delta_{3}}{\delta_{2}+\delta_{3}} \\
q_{n} & =q\left(\delta_{n+1}-\delta_{n+2}\right), n=1,2,3 \bmod (3) \\
\tau_{n} & =-\tau+\delta_{n} \xi
\end{aligned}
$$

For a given choice of the characteristic linear velocities in Eqs. (1), the trappon solution of Eqs. (8) is completely determined by just three independent real parameters, namely $p, q, a$ (with the restrictions that $p>0, a>0$ and $a^{2}>p^{2}$ ). The parameter $p$ is associated with the rescaling of the wave amplitudes, and of coordinates $\tau$ and $\xi$. Whereas $a$ measures the amplitude of the CW background in wave $A_{1}^{T}$. The parameter $q$ adds a phase shift which is linear in both $\tau$ and $\xi$.

The trappon solution (8) extends in time from $\tau=-\infty$ to $\tau=+\infty$ and at large distances (i.e., for $\xi \rightarrow+\infty$ ) it exhibits a time-periodic behavior. Nevertheless, as we shall see in next section, Eqs. (8) exactly describe the mixing of the ZS soliton (6) with the quasi-CW (5) background signal over the finite interval $\left[\tau_{i}, \tau_{f}\right]$ (which correspond to the times of turning on and off the $\mathrm{CW}$ wave at frequency $\omega_{1}$, respectively).
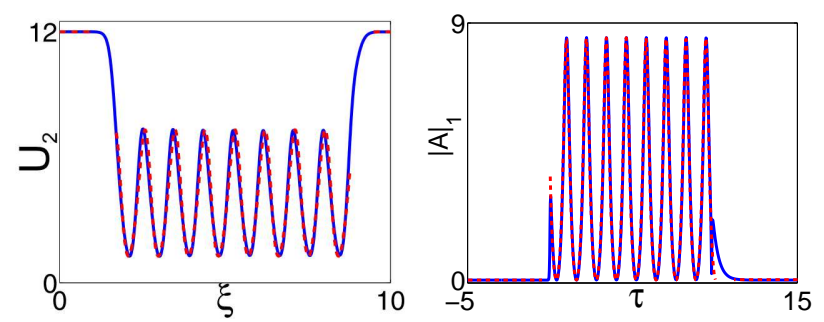

Fig. 2. Numerical evolution (continuous lines) and theoretical prediction from Eqs. (8) (dotted lines) of the energy at $\omega_{2}$ and of the output pulse profile at $\omega_{1}$ for the case of Fig. 1.

\section{ThrEe-WAVE ADIABATIC SOLITON SHAPING}

Indeed, one may analytically predict the characteristic parameters $p, q, a$ of the generated TWI trappon from the corresponding $P, \phi$ parameters of the isolated input $\mathrm{ZM}$ soliton, and the complex amplitude $C$ and the frequency shift $\gamma$ of the $\mathrm{CW}$ signal. In order to demonstrate this, it is sufficient to suppose that the input ZM soliton adiabatically (i.e., without emission of radiation) reshapes into a trappon soliton after its collision with the quasi-CW background at a given point in time (say, at $\tau=\tau_{i}$ ). In the spectral domain [16] this hypothesis is equivalent to imposing that the input $\mathrm{ZM}$ soliton, after the collision with the $\mathrm{CW}$ background, transfers its discrete eigenvalue to the trappon soliton. In the frame of this basic hypothesis, the conservative nature of the threewave interaction permits us to suppose that: i) the energy $U_{23}$ (4) of the input TWI soliton is conserved in the generated trappon soliton; ii) the phase of the $\omega_{2}$ frequency components of the input TWI soliton and of the generated TWI soliton varies in a continuous manner across their time interface (i.e., at $\tau=\tau_{i}$ ); iii) the amplitude and the phase of the $\mathrm{CW}$ background coincide with the corresponding values of the asymptotic plateau of the component at frequency $\omega_{1}$ of the generated TWI trappon. The above three conditions, after some straightforward calculations, permit us to obtain the following equations that relate the parameters of the incident and of the transmitted solitons (i.e., before and after the collision, respectively)

$$
P=p, \quad a=|C| / \sqrt{\delta_{2} \delta_{3}}, \quad q\left(\delta_{2}-\delta_{3}\right)=\phi,
$$

with the restrictions that $|C|^{2}>P^{2} \delta_{2} \delta_{3}$ and $\gamma=\phi$. The above conditions define the matching relations among the amplitudes and the phases of both the input ZM soliton and of the CW signal which should be satisfied in order to excite the trapponic soliton. As an example, consider the case of Fig. 1, where the $\mathrm{CW}$ background control is described by Eqs. (5) with $C=3$, $\gamma=1$, and the input ZM soliton (6) is obtained from Eqs. (6) with $P=1.5, \phi=1$. After the collision with the $\mathrm{CW}$ background, Eqs. (10) predict that the generated trappon is obtained from Eqs. (8) with $p=1.5, q=1$, and $a=3 / \sqrt{2}$. We confirmed the accuracy of this prediction by means of extensive comparisons of the analytical expression (8) with numerical solutions of the TWI Eqs. (1). For example, Fig. 2 displays both numerically computed and analytical evolutions 
a)

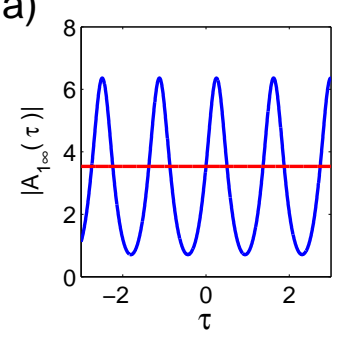

c)

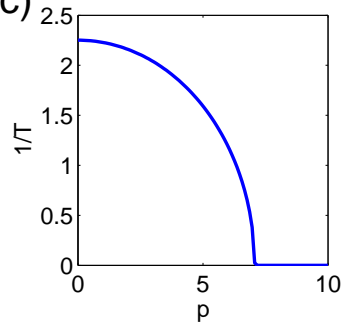

b)

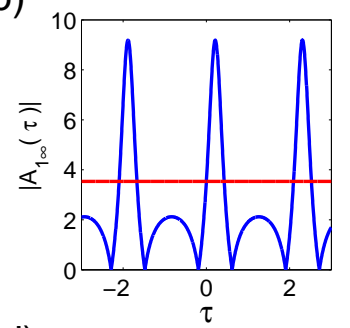

d)

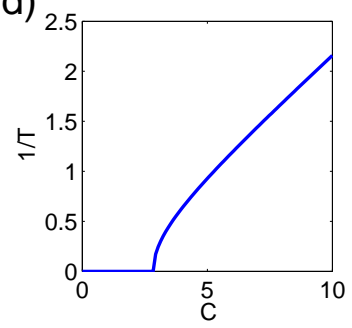

Fig. 3. Output pulse train (11) amplitude (blue line) and its time-average value (red line) for: a) $C=2.5 \sqrt{2}, P=1$ and b) $C=2.5 \sqrt{2}, P=2$; Pulse train frequency $f=1 / T$ c) vs. $P(C=10)$; and d) vs. $C(p=2)$.

for the energy $U_{2}$ (along the crystal length $\xi$ ) and for the output transverse profile of wave $A_{1}$ (along $\tau$ ) (these correspond to the same interaction process previously shown in Fig. 1).

\section{CONTROlled PUlse TRAin GENERATION}

The amplitude profile of the generated train of ultrashort pulses at frequency $\omega_{1}$ may be exactly described by performing the limit for $\xi \rightarrow+\infty$ of the first of Eqs. (8), which yields

$\left|A_{1 \infty}^{T}(\tau)\right|=|C|\left|1-4 \cos (\beta) \frac{\sin (\pi \tau / T) \sin (\pi \tau / T-\beta)}{1-\cos (\beta) \cos (2 \pi \tau / T-\beta)}\right|$

where $|C|=a \sqrt{\delta_{2} \delta_{3}}$ (see (10)), $\tan (\beta)=\sqrt{a^{2}-p^{2}} / p$ (see (9)) and period $T=\pi / \sqrt{a^{2}-p^{2}}$. Two different examples of the generated pulse trains as described by Eqs.(11) are shown in Fig. $3 a)-b)$.

Equation (11) provides relatively simple expressions that relate the parameters of the injected soliton pulse and $\mathrm{CW}$ background to the main features of the generated output pulse train. Indeed, $\left|A_{1 \infty}^{T}\right|$ is a time periodic signal (with period $T$ ) with average value approximatively equal to $|C|$. Moreover, from Eqs.(11) one obtains that the peak amplitude of the generated pulses is $M=|C|+2 \sqrt{\delta_{2} \delta_{3}} P$; whereas the minimum amplitude is $m=|C|-2 \sqrt{\delta_{2} \delta_{3}} P$. Hence the maximum intensity contrast for the generated train is obtained for $|C|=2 P \sqrt{\delta_{2} \delta_{3}}$ : in this case, the field amplitude at the minima vanishes and the peak amplitudes reads as $M=4 \sqrt{\delta_{2} \delta_{3}} P$.

In Figure $3 \mathrm{c}$ )-d) we illustrate the analytically predicted dependence of the repetition rate of the ultra-short pulse train $\mathbf{R}=\mathbf{1} / \mathbf{T}=\sqrt{|\mathbf{C}|^{2} /\left(\delta_{\mathbf{2}} \delta_{\mathbf{3}}\right)-\mathbf{p}^{2}} / \pi$, as a function of the amplitudes of the input short pulse and of the $\mathrm{CW}$ signal, respectively. As it can be seen in Fig. 3 c), the repetition

rate is the largest whenever the amplitude of the input soliton vanishes. Whereas there is a critical input soliton amplitude, above which the period of the generated pulse train diverges to infinity. On the other hand, Fig. 3 d) shows that when varying the $\mathrm{CW}$ background amplitude, a finite repetition rate is only obtained for amplitudes $|C|$ above a certain threshold level, and the rate grows larger with $|C|$ in a linear fashion.

The above results show that the peak amplitude, the time width and the repetition rate of the pulse train which is generated at $\omega_{1}$ may be controlled in a stable manner by simply adjusting the intensity level of the input $\mathrm{CW}$ signal $|C|$ and/or the amplitude level (hence the time width) $P$ of the input short soliton pulse.

It is worth noting the efficiency of the proposed method for generating pulse trains, since theoretically the entire energy of the pump pulse $A_{1}$ is converted into the train. Indeed, from the energy conservation relation (3) and the fact that at the end of the interaction $A_{3}=0$, it follows that the energy of the quasi-CW pulse is conserved before and after the collision with the control pulse $A_{2}$.

It should be pointed out that the generation process we have so far described may be thought of as a way of "writing" a pulse train on a quasi $\mathrm{CW}$ signal. The opposite process of "erasing" such wave train to go back to a flat, quasi CW, signal is also possible. Indeed, it is easy to realize that this reverse process is well described in a similar way by the formation of the trappon soliton solution of the TWI Eqs. (1) which is obtained from the expression (8) via the invariance transformation $\xi \rightarrow-\xi, \tau \rightarrow-\tau, A_{1} \rightarrow A_{1}, A_{2} \rightarrow A_{2}, A_{3} \rightarrow-A_{3}$.

We would like to point out that the observation of the above described three-wave phenomena appears to be readily achievable in nonlinear optical experiments. For instance, let us consider the eee interaction of three-waves with carrier wavelengths of $\lambda_{1}=1.55 \mu \mathrm{m}, \lambda_{2}=3.4 \mu \mathrm{m}, \lambda_{3}=1.064 \mu \mathrm{m}$ in a $8 \mathrm{~cm}$ long periodically poled bulk Lithium Tantalate crystal with $28 \mu \mathrm{m}$ periodicity. In this case, the parametric mixing of a $200 \mathrm{fs}$ incident pulse with a quasi-CW signal (say, with a $3 p s$ time duration) leads to the generation of a train of $250 \mathrm{fs}$ pulses with a repetition rate of about $2 \mathrm{THz}$; the corresponding peak power levels of the incident and of the generated fields are of the order of a few hundreds of $M W / \mathrm{cm}^{2}$. In practical terms, the upper limit to the maximum repetition rate of the generated pulse train which can be achieved by the present technique will be set by the limit of validity of Eqs. (1), which hold true in three-wave physical systems whenever the presence of group velocity dispersion within each wave can be neglected.

\section{Conclusions}

We revealed that the three-wave parametric interaction of an ultra-short ZM soliton pulse with a quasi-continuous wave $(\mathrm{CW})$ signal in a weakly quadratic nonlinear and dispersive medium may lead to the generation of a trapped soliton, or trappon. Moreover, we predicted that the excitation of trapped solitons may lead to a new class of optically controlled, cavityless sources of ultra-short pulse trains with up to multi-THz repetition rates. 


\section{ACKNOWLEDGEMENTS}

The present research of A.D. and S.W. was carried within the French CNRS project GDR 3073.

\section{REFERENCES}

[1] X. Zhang, A.L. Lytle, T. Popmintchev, X. Zhou, H.C. Kapten, M.M. Murnane, and O. Cohen, "Quasi-phase-matching and quantum-path control of high harmonic generation using counterpropagating light," Nat. Phys., vol.3, no.4, pp. 270-275, May 2007.

[2] A.M. Weiner, D.E. Leaird, G.P. Wiederrecht, and K.A. Nelson, "Femtosecond pulse sequences used for optical manipulation of molecular motion," Science, vol. 247, no. 4948, pp. 1317-1319, Mar. 1990.

[3] D. Umstadter, E. Esarey, and J. Kim, "Nonlinear plasma waves resonantly driven by optimized laser pulse trains," Phys. Rev. Lett., vol. 72, no. 8, pp. 1224-1227, Feb. 1994.

[4] Y. Liu, S.G. Park, and A.M. Weiner, "Enhancement of narrow-band terahertz radiation from photoconducting antennas by optical pulse shaping," Opt. Lett., vol. 21, no.21 pp. 1762-1764, Nov. 1996.

[5] P. Petropoulos, M. Ibsen, M.N. Zervas, and D.J. Richardson, "Repetition frequency quadruplication through Fabry-Perot filtering," Opt. Lett., vol. 25, no. 8, pp. 521-523, Apr. 2000.

[6] K. Yiannopoulos, K. Vyrsokinos, E. Kehayas, N. Pleros, K. Vlachos, H. Avramopoulos, and G. Guekos, "Repetition frequency quadruplication through Fabry-Perot filtering," IEEE Photon. Technol. Lett., vol. 15, no. 9, pp. 1294-1296, Sep. 2003.

[7] S. Longhi, M. Marano, P. Laporta, O. Svelto, M. Belmonte, B. Agogliati, L. Arcangeli, V. Pruneri, M.N. Zervas, and M. Ibsen, "40-Ghz pulse-train generation at 1.5 um with a chirped fiber gratings as frequency multiplier," Opt. Lett., vol. 24, no. 19, pp. 1481-1483, Oct. 2000.

[8] J. Azana, and M. Muriel, "Temporal self-imaging effects: theory and application for multiplying pulse repetition rates," IEEE J. Sel. Top. Quantum Electron., vol. 7, no. 4, pp. 728-744, Jul.-Aug. 2001.

[9] J. Caraquitena, Z. Jiang, D.E. Leaird, and A.M. Weiner, "Tunable pulse repetition-rate multiplication using phase-only line-by-line pulse shaping," Opt. Lett., vol. 32, no. 6, pp. 716-718, Mar. 2007.

[10] A. Hasegawa, "Generation of a train of soliton pulses by induced modulation instability in optical fibers," Opt. Lett., vol. 9, no. 7, pp. 288290, 1984

[11] S. Coen, and M Haelterman, "Continuous-wave ultrahigh repetition rate pulse train generation through modulation instability in a passive fiber cavity," Opt. Lett., vol. 26, no.1, pp. 39-41, Jan. 2001.

[12] S. Pitois, J. Fatome, and G. Millot, "Generation of a 160-Ghz transform limited pedestal free pulse train thorough multiwave mixing compression of a dual-frequency beat signal," Opt. Lett., vol. 27, no. 19, pp. 1729-1731, Oct. 2002.

[13] T. Inoue, J. Hiroishi, T. Yagi, and Y. Mimura, "Generation of in-phase pulse train from optical beat signal," Opt. Lett., vol. 32, no. 11, pp. 15961598, Jun. 2007.

[14] C. Conti, G. Assanto, and S. Trillo, "Cavityless oscillation through backward quasi-phase-matched second-harmonic generation," Opt. Lett. vol. 24, no. 16, pp. 1139-1141, Aug. 1999.

[15] M. Conforti, A. Locatelli, C. De Angelis, A. Parini and S. Trillo, "Selfpulsing instabilities in backward parametric wave-mixing," J. Opt. Soc. Am. B, vol. 22, no. 10, pp. 2178-2184, Oct. 2005.

[16] V. E. Zakharov and S. V. Manakov, "Resonant interaction of wave packets in non-linear media," Sov. Phys. JETP Lett., vol. 18, no. 7, pp. 243-245, Oct. 1973.

[17] A. Degasperis, Solitons, boomerons, trappons, in: F. Calogero (Ed.), Nonlinear Evolution Equations Solvable by the Spectral Transform, Pitman, London, 1978, pp. 97-126.

[18] F. Calogero and A. Degasperis, "Novel solutions of the system describing the resonant interactions of three waves," Physica D vol. 200, no. 3-4, pp. 242-256, Jan. 2005.

[19] A. Degasperis, M. Conforti, F. Baronio and S. Wabnitz, "Stable control of pulse speed in parametric three-wave solitons," Phys. Rev. Lett., vol. 97, no. 9, p. 093901, Sep. 2006.

[20] M. Conforti, F. Baronio, A. Degasperis and S. Wabnitz, "Parametric frequency conversion of short optical pulses controlled by a CW background," Opt. Express, vol. 15, no. 19, pp. 12246-12251, Sep. 2007.

[21] M. Conforti, F. Baronio, A. Degasperis and S. Wabnitz, "Inelastic scattering and interactions of three-wave parametric solitons," Phys. Rev. $E$, vol. 75, no. 6, p. 065602(R), Dec. 2006.
Fabio Baronio was born in Brescia (Italy). In March 2001, he received the Laurea degree (BS, MS) in Electronic Engineering (summa cum laude) from the University of Brescia, Italy. In March 2005 he received the $\mathrm{PhD}$ degree in Electronic and Telecommunication Engineering from the University of Padua, Italy. From March 2005 he is an Assistant Professor in Electromagnetic Fields at the University of Brescia. The scientific research activity of Fabio Baronio deals with Electromagnetism, Photonics and Telecommunications. $\mathrm{He}$ has authored or co-authored of over 50 refereed papers and conference presentations. He is a member of the Optical Society of America and of the IEEE Laser and Electro-Optics Society.

Matteo Conforti was born in Brescia, Italy, in 1978. He received the Laurea degree and the $\mathrm{Ph} . \mathrm{D}$. degree in electronic engineering from University of Brescia, Brescia, in 2003 and 2007, respectively. Currently he is Research Fellow at the University of Brescia. His main research interests include nonlinear optics and numerical methods for electromagnetism. He is the author and coauthor of over 30 refereed papers and conference presentations.

Antonio Degasperis obtained the degree "Laurea in Fisica" from the University of Rome "La Sapienza" in 1964. He is presently full professor of Mathematical Methods of Physics and Theoretical Physics in the Department of Physics of this University. He formerly acted as Chairman of the Department Theoretical Group in Rome, and as member of the Research Funding Committee of the Italian Ministry of Education and Research. He is reviewer for several international journals in the area of wave scattering and soliton theory and has been coeditor of few journals (Inverse Problems, Nonlinearity, EuroPhysics Letters); he is presently Scientific Advisor for EuroPhysics Letters. His present research interests are in integrable models of nonlinear wave propagation, mainly in one- and two- dimensional spaces (soliton theory). Author (and co-author) of more than 100 refereed articles and conference contributions, he has co- authored one book published by North-Holland, and has co-edited several conference proceedings.

Stefan Wabnitz (M03) received the Laurea degree in electronics engineering from the University of Rome La Sapienza, Rome, Italy, the M.S. degree in electrical engineering from the California Institute of Technology (Caltech), Pasadena, and the Ph.D. degree in applied electromagnetism from the Italian Ministry of Education, Rome. He has been a Researcher with the Optical Communications Department, the Ugo Bordoni Foundation, between 1985 and 1996, where he has contributed the theory of nonlinear wave propagation in optical fibers and waveguides, with particular interest in wave instabilities and soliton phenomena. In 1996, he became a Full Professor in physics with the Université de Bourgogne, Dijon, France. Between 1999 and 2003, he was on leave from the University to carry out research and development in the telecommunication industry. He spent two years working on optical soliton effects for submarine system applications at the Alcatel Research and Innovation Laboratory, Marcoussis, France, and two years as Manager of the Advanced Technology Group, Xtera Communications, Allen, TX. Since 2007 he joined as full professor the department of electronics for automation at the University of Brescia. His current research activities involve nonlinear propagation effects in high-bit-rate optical communication systems and in photonic crystal structures. Dr. Wabnitz is a member of the Optical Society of America and of the IEEE Laser and Electro-Optics Society. 